\title{
Three dimensional reconstruction of cross sectional intracoronary ultrasound: clinical or research tool?
}

Carlo Di Mario, Clemens von Birgelen, Francesco Prati, Bobby Soni, Wenguang Li, Nico Bruining, Peter P T de Jaegere, Pim J de Feyter, Patrick W Serruys, Jos R T C Roelandt

Cardiac
Catheterisation and
Intracoronary
Imaging Laboratory,
Division of
Cardiology,
Thoraxcenter,
Erasmus University
Rotterdam, The
Netherlands
C Di Mario
C von Birgelen
F Prati
N Bruining
P P T de Jaegere
P J de Feyter
P W Serruys
J R T C Roelandt
Department of
Biomedical
Engineering,
University Hospital
Rotterdam-Dijkzigt
and Erasmus
University,
Rotterdam, The
Netherlands
W Li
Research and
Development
Department, Indec
Medical Systems,
Mountain View, CA,
USA
B Soni
Correspondence to:
Dr J T C Roelandt,
Division of Cardiology,
Thoraxcenter BD 408,
University Hospital Dijkzigt,
PO Box 1738 DR
Rotterdam, The
Netherlands.

Imaging Laboratory,

Thoraxcenter,

Erasmus University

Rotterdam, The

C von Birgelen

F Prati

N Bruining

P J de Feyter

$P$ W Serruys

J R T C Roeland

ent of

University Hospital

University,

Rotterdam, The

Research and

Developmen

Mountain View, CA

USA

Thoraxcenter BD 408,

PO Box 1738 DR

Netherlands.

In a recent survey conducted in 30 centres with large experience in intracoronary ultrasound, three dimensional reconstruction of intracoronary ultrasound was ranked as the technical development with the lowest priority, ${ }^{1}$ suggesting that it is perceived by most users of intracoronary ultrasound as a research tool with limited clinical relevance. However, although cross sectional ultrasound provides unique information on lumen and wall pathology, it cannot determine the spatial relationships among multiple ultrasonic cross sections. The need to examine vessel architecture longitudinally explains the many efforts to develop methods of three dimensional reconstruction of intracoronary ultrasound images. ${ }^{23}$

In this paper we present the steps required for three dimensional reconstruction of intracoronary images and the methods of display and quantitative analysis. Limitations of the technique and its current and future clinical potential are discussed.

\section{Three dimensional reconstruction: technique}

Three dimensional reconstruction requires the acquisition of the basic ultrasonic images and segmentation of the digitised images, two steps which greatly influence the final result.

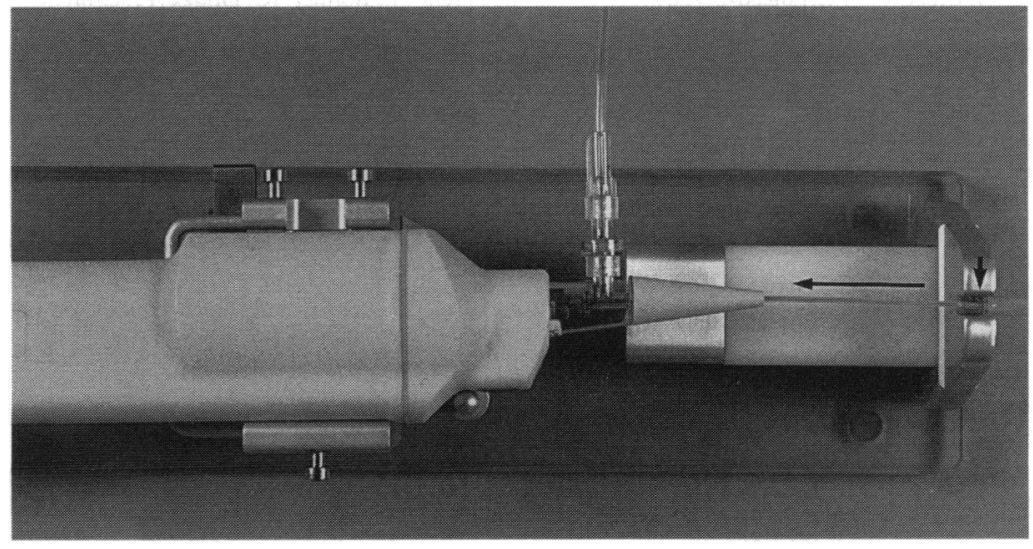

Figure 1 The motor unit of the ultrasound catheter, connected to the proximal end of the imaging cable, is mounted on a handle of the motorised pull back device. Since the proximal end of the external catheter sheath is fixed (arrowhead), the motion of the handle induces a pull back of the telescopic imaging cable inside the external sheath at a constant speed (direction indicated by the arrow). (MicroView 2.9 ultrasound catheter, Sunnyvale, CA, USA.)
IMAGE ACQUISITION

Three alternative methods can be used: (1) continuous pull back at a constant speed; (2) a catheter displacement sensing device; and (3) an ECG gated motorised pull back or image acquisition.

Continuous pull back at a constant speed

The images are acquired in the form of "slices" during a slow pull back at a constant speed, permitting axial measurements during the image acquisition, since the length of the reconstructed segment is defined by the time interval between two cross sections multiplied by the pull back speed. ${ }^{4}$ Recent improvements in catheter design have facilitated the use of a motorised pull back device. An echo transparent distal sheath can accept a guidewire for intracoronary insertion or alternatively a flexible rotating imaging cable (fig 1 ), avoiding sudden changes in pull back speed. The small dimension of the sheath imaging cable assembly (distal external diameter $=1 \mathrm{~mm}$ ) allows repeated assessment of the arterial segment. The pull back speed is a compromise between the resolution of the longitudinal reconstruction and the need to avoid an excessive duration of the examination procedure and subsequent reconstruction. In practice, a pull back speed of between 1 and 0.5 $\mathrm{mm} / \mathrm{s}$ is used. A pull back time of 30 to 60 seconds is then required to examine a $3 \mathrm{~cm}$ long vessel, generally comprising the coronary stenosis and the adjacent reference segments. Sampling must be performed using optimal machine settings and in particular a constant zoom factor.

\section{Catheter displacement sensing device}

A disposable sensor, usable with all ultrasound catheters, has been developed at the Thoraxcenter. The linear motion of the catheter against a roller connected to the arterial sheath or $\mathrm{Y}$ piece connector is converted into an electronic signal. This approach permits continuous measurement of the depth of the catheter during manual or motorised pull backs and displays it on the video screen, yielding accurate and reproducible results. ${ }^{5}$ Movement of the guiding catheter during insertion or pull back of the ultrasound catheter is a serious reason for concern in intracoronary application. The system, however, can be connected to the telescopic proximal end of the last generation ultrasound 
catheter in order to measure the depth of insertion of the imaging core into the distal sheath.

\section{ECG gated motorised pull back or image acquisition}

Systolic-diastolic movement of the imaging catheter and cyclic changes of the vessel dimensions are major limitations for accurate three dimensional reconstruction of lumen and vessel wall, suggesting consistent use of end diastolic images for quantification. ${ }^{6}$ An ECG gated three dimensional reconstruction can be obtained by using a stepping motor and a dedicated acquisition station. At the Thoraxcenter we use the commercially available Echoscan (TomTec, Munich, Germany) ${ }^{7}$ which controls the stepping motor of the pull back device using steering logic which takes account of the variation of the heart cycle. Before acquisition, variables such as the distance between consecutive transducer positions (step resolution) and the length of the segment studied (scan distance) are defined. The acquisition is started from the peak of the $R$ wave and images are grabbed during the following cardiac cycle at a speed of 25 frames per second. After this sequence the system determines whether an individual $R-R$ interval fits in the predefined range. If the requirements are fulfilled, the digitised frames are stored and the catheter is withdrawn up to the next transducer position. Otherwise, the frames are rejected and a new sequence of images is acquired at the same transducer position, starting at the next $R$ wave (fig 2). Images of successive cardiac cycles are acquired until the end of the scan distance is reached. The advantage of such an ECG gated image acquisition and pull back is that the three dimensional reconstruction can be shown in a cine loop format, enabling the dynamic visualisation of the coronary artery.

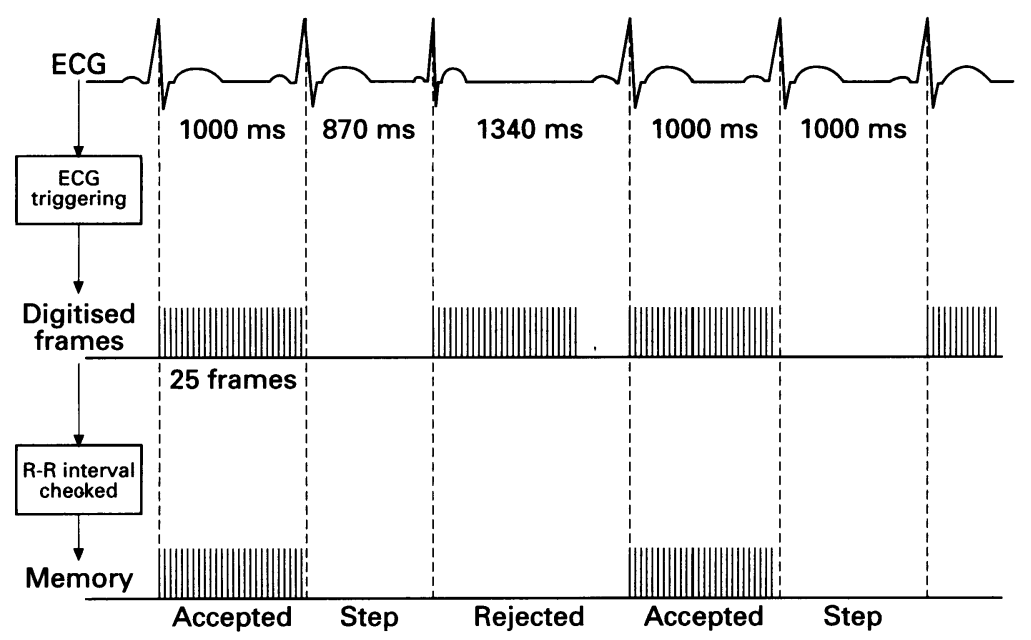

$R-R$ interval $=1000 \mathrm{~ms} \pm 100 \mathrm{~ms}$ Respiration gating $=$ OFF

Figure 2 Schematics of the ECG gated acquisition during motorised pull back with a dedicated stepping motor unit. Only the images obtained during cardiac cycles of predetermined length are accepted, triggering a further movement of the catheter.
IMAGE SEGMENTATION

The subsequent essential step after image acquisition and digitisation is the image segmentation which distinguishes between the blood pool and structures of the vessel wall in all the digitised ultrasound images. The high frequencies ( $\geqslant 20 \mathrm{MHz}$ ), required to achieve high resolution imaging of the vessel wall, induce a high echogenicity of the blood pool which impairs the automated detection of the lumen-intima boundary. A "hard" threshold cannot be successfully applied in order to eliminate this interference because of the intensity overlap between the backscatter intensity of blood pool and vessel wall.

The backscatter pattern of flowing blood cells shows a speckled texture which varies over time, whereas the echo signal of the vessel wall shows a more fixed pattern. The blood speckle identification algorithm can be based on this principle. The blood pool is identified using statistical pattern recognition and the pixels identified as blood are removed. An immediate appreciation of the accuracy of blood subtraction is facilitated by the possibility of colour encoding the vessel lumen displayed in red. User interaction is allowed to modify pattern recognition variables. Finally, manual correction of the individual cross sectional luminal contours can be performed. An advantage of this method is that no assumptions on the lumen geometry are required, thus allowing segmentation of irregular lumen shapes such as may occur after coronary interventions. Recently this method has been validated in normal rabbit aortas. ${ }^{8}$

An alternative segmentation approach is the contour detection based on the application of a minimum cost algorithm that will be discussed later.

THREE DIMENSIONAL RECONSTRUCTION Full grey scale volume rendering has replaced previous methods for three dimensional reconstruction of intracoronary ultrasound images. The vessel can be displayed using a variety of computer generated two dimensional (transverse, longitudinal, oblique) or true three dimensional images such as lumen casts or cylindrical image formats which are cut lengthwise and opened (clam shell view), showing the intimal surface of the vessel.

\section{ON LINE RECONSTRUCTION AND QUANTIFICATION: THORAXCENTER EXPERIENCE}

At the Thoraxcenter the EchoQuant system (Indec, Sunnyvale, CA, USA) is used for on line three dimensional reconstruction, display, and quantitative analysis of intravascular ultrasound images. The system operates on a dedicated Intell Pentium $60 \mathrm{MHz}$ personal computer using the OS/2 operating system, digitising the analogue video image on line or off line with a digitisation frame rate of 8.5 images per second. During the image acquisition (maximum 255 slices) the reconstruction of the longitudinal section is started and immediately displayed on the screen. The vessel length traversed depends on the pull back 


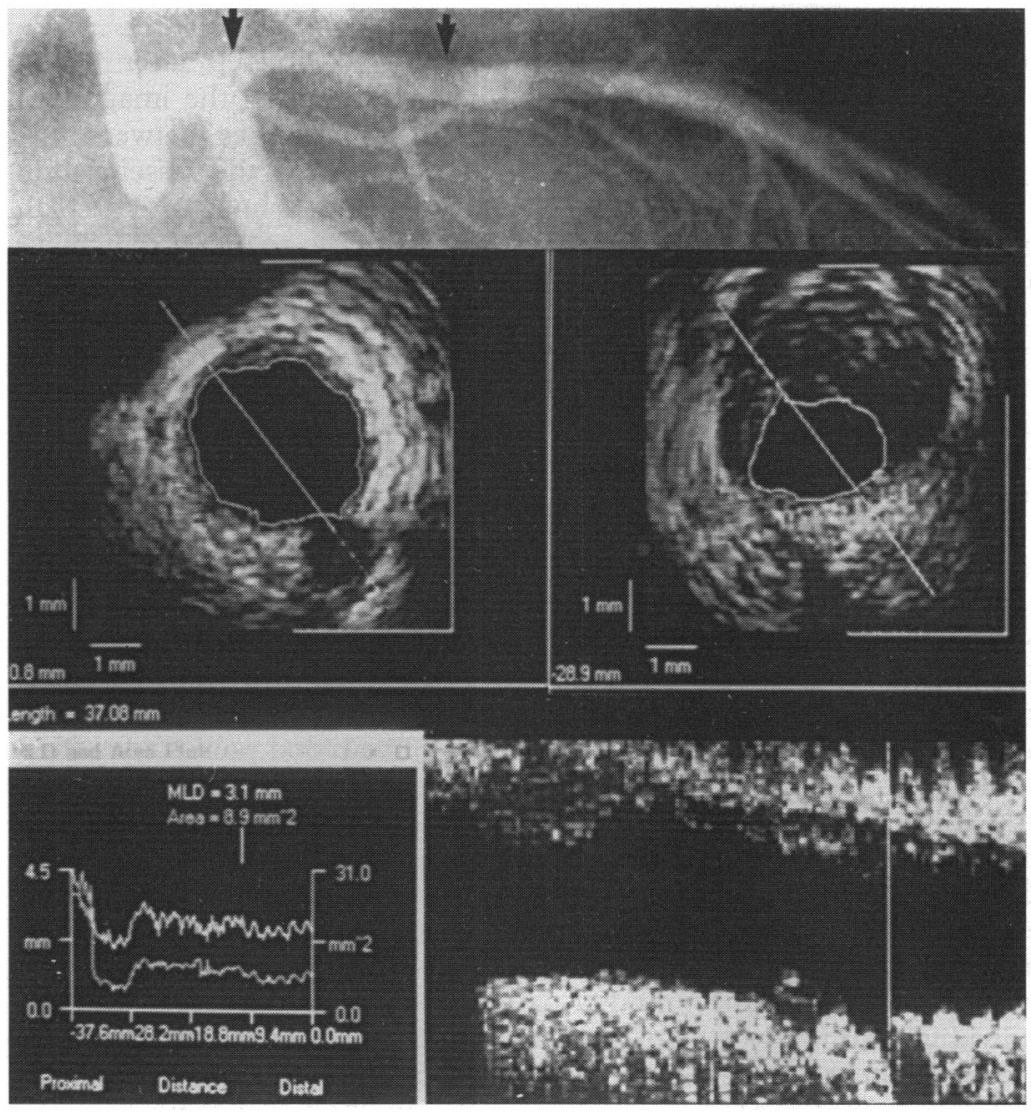

Figure 3 Lower right panel: longitudinal view of a left anterior descending coronary artery before directional coronary atherectomy. A soft large plaque is visible in the proximal segment of the vessel (left side). A side branch, indicated by a vertical line and visible also in the transverse view (mid-left panel), can be followed for the first millimetres from its origin and corresponds to the first septal branch visible in the angiogram (upper panel). origin and corresponds to the first septal branch visible in the angiogram (upper
The side branch is also visible in the transverse view in the mid-left corner. The arrowheads in the angiogram indicate the reconstructed segment. In the left lower panel the corresponding measurements of cross sectional area (Area, lower line) and minimum luminal diameter (MLD, upper line) are shown. The arrowheads in the angiogram indicate the length of the reconstructed segment. In the mid-right panel, a transverse view at the level of the plaque is displayed. Note that the maximum thickness of the plaque is not perpendicular to the side branch, suggesting an orientation of the cutter different from the orientation indicated by the angiogram. The lumen-plaque interface has been automatically detected and displayed in both transverse views.

speed; thus using a pull back speed of $1 \mathrm{~mm} / \mathrm{s}$ a $30 \mathrm{~mm}$ long vessel segment can be reconstructed.

In the EchoQuant system a specialised form of ray casting is employed for the reconstruction of sequential arterial cross sections. The short time required for processing the 255 slices and reconstructing the three dimensional image (120 seconds using the above described hardware) allows the use of the system for clinical decision making in the catheterisation laboratory. During the segmentation process identifying the blood pool, the cross sectional images are presented successively in the left upper quadrant of the screen and the longitudinal view is shown in the lower panel and repeatedly updated. The longitudinal view is particularly suitable for the assessment of the length of a stenosis or a dissection (fig 3). A diagram showing measurements of cross sectional area and of minimum lumen diameter over the entire reconstructed segment is automatically generated and the lumen-wall contours are displayed in all the transverse views. Finally, the three dimensional image is presented in a cylindrical format, opened longitudinally with both halves tilted back 30 degrees. The image can be animated by a rotation perpendicular to the long axis of the vessel in order to display all the details of the intimal surface (fig 4).

The reconstructed images can be exported to a general purpose three dimensional software package for further processing and image display (Micro Voxel, Indec, CA, USA).

OFF LINE RECONSTRUCTION AND

QUANTITATIVE ANALYSIS

The quantitative package described above is unable to detect the blood-intima interface properly in suboptimal images and does not provide an approach for automated detection of the external contour of the vessel. The measurement of plaque area is of paramount importance for the study of atherosclerosis and the results of interventions. At the Thoraxcenter the research efforts have been concentrated in this field, and an analysis system has been developed that provides quantification of lumen and plaque dimensions, based on the application of an algorithm previously described and validated in vitro and in vivo. ${ }^{9-11}$

This system allows the acquisition of 200 cross sectional images so that a segment of $2 \cdot 0$ $\mathrm{cm}$ can be analysed and reconstructed at a pull back speed of $1 \mathrm{~mm} / \mathrm{s}$ with high longitudinal resolution. Two perpendicular longitudinal views are reconstructed from the series of cross sectional images. A minimum cost algorithm is applied for automated detection of the intimal leading edge and the external contour of the vessel. Operator interaction is allowed to indicate one or more points in the longitudinal images to be used as fixed constraints for the contour detection process. Four individual edge points, derived from the contours on the longitudinal images, are used as constraints guiding the final contour detection in all the individual cross sectional images (fig 5). The accuracy of the contour detection can be visually checked and manual correction may be performed. ${ }^{12}$ The cross sectional image with the minimum luminal cross sectional area is automatically defined. Areas of lumen, total vessel, and plaque are measured for each frame and the corresponding mean diameters are calculated. The intra-observer variability of the described method for the measurements of lumen and plaque volume was assessed in vivo in 10 atherosclerotic coronary arterial segments with a maximum area stenosis ranging from $43 \cdot 8 \%$ to $83.4 \%$. A high reproducibility of the volumetric measurements of plaque and lumen was observed with mean signed differences between $-0.6 \%$ and $0.4 \%$ and standard deviations not exceeding $2 \cdot 6 \% .^{12}$

\section{Three dimensional reconstruction: clinical applications}

ASSESSMENT OF LUMEN AND PLAQUE VOLUME Intracoronary ultrasound provides detailed 

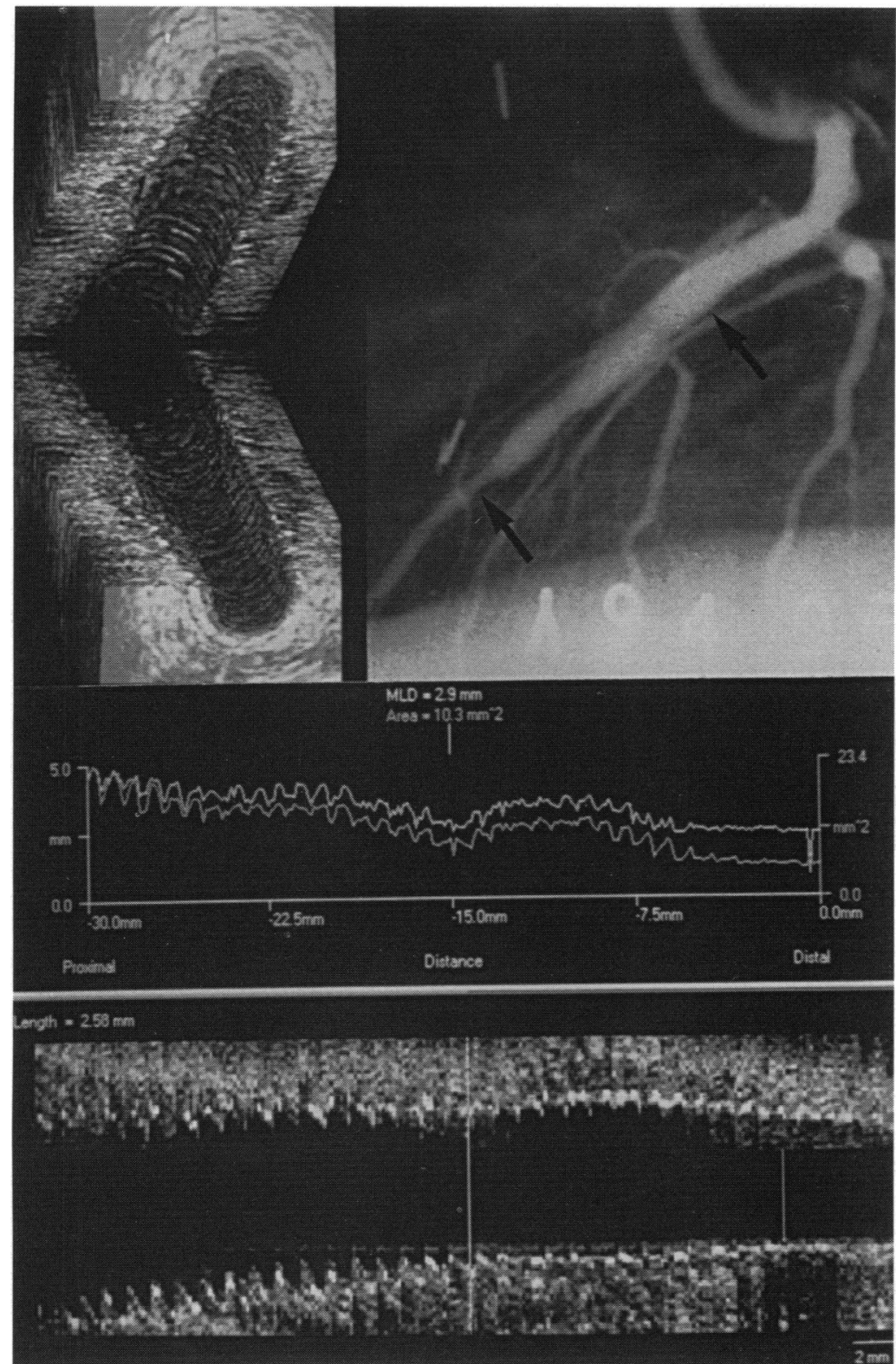

Figure 4 Right upper panel: cineangiogram of a left anterior descending coronary artery 3 months after implantation of a Wallstent (Schneider, Bulach, Switzerland). Black arrows indicate the segment reconstructed with three dimensional intracoronary ultrasound. Left upper panel: a cylindrical reconstruction of that artery is cut open and presented in a clam shell view. In the distal end (towards the observer), the echogenic wires of the stent are visible, with the presence of a moderate intimal thickening inside the stent in the more distal part. Lower panels: longitudinal view and corresponding area function (lower line) and diameter function (upper line) of the reconstructed segment. The progressive tapering of the vessel and the distal narrowing of the coronary lumen are clearly displayed and quantified. Note the saw fish appearance of the diameter function in the proximal segment induced by the systolic expansion of the vessel.

tomographic information about coronary lumen and plaque which are not available with angiography. ${ }^{13}{ }^{14}$ Rosenfield et $a l^{15}$ have proposed the application of automated edge detection algorithms for the analysis of a three dimensional lumen cast. With this method a rapid on line assessment of the minimum cross sectional area before and after interventions on peripheral arteries was possible. Matar et $a l^{16}$ used a motorised pull back handle to obtain a uniform distance between consecutive cross sections in the examination of arterial specimens in vitro and of human coronary arteries in vivo. The volumes of the reconstructed lumen correlated well with the histol- ogy measurements and with the results of biplane quantitative angiography.

The measurement of plaque volume allows a direct assessment of the changes of vascular dimensions induced by pharmacological or dietary therapy aiming at regression of atherosclerosis ${ }^{1718}$ and by interventional procedures. Galli et al $^{18}$ compared the true plaque volume of a vessel phantom and the measurements based on planimetry of consecutive cross sections with the results of direct three dimensional reconstruction. Plaque volumes measured by three dimensional reconstruction overestimated the true plaque volume of the phantom while more accurate measurements were obtained from direct planimetry of the echographic cross sections. The importance of the algorithm used for image segmentation is documented by the accurate measurements obtained by Hausmann et al in normal rabbit aortas ${ }^{8}$ and by von Birgelen et al in vessel phantoms. ${ }^{12}$

The application of computerised systems of automated edge detection for adventitial and lumen border ${ }^{12}$ has the potential to increase the reproducibility of the measurements of plaque and lumen volume $(r=0.99$ and $r=$ 0.97 respectively) and the correlation of lumen volume measurements obtained during subsequent independent pull back data sets $(r=0.99) .{ }^{19}$

\section{ASSESSMENT OF INTERVENTIONS}

Rationale

The accurate detection of vessel dimension and plaque characteristics provided by intracoronary ultrasound can be used to select type and size of device to be used and to guide the interventions. On line three dimensional reconstruction offers practical advantages in comparison with a conventional ultrasound examination. The on line longitudinal view of the stenotic segment before dilatation provides immediate information on the length of the stenosis and on the diameter of the lumen and total vessel in the reference segment. Based on this information, the length and diameter of balloon or device to be used can be more easily selected. The comparison between different cross sections (that is, reference and stenosis) can be performed by simply moving a cursor and does not require repeated insertions of the ultrasound probe or time consuming reviews of the video recordings. The effects and mechanisms of interventions and of restenosis ${ }^{19-25}$ can be studied using a rigorous comparison of corresponding cross sections, provided that the pull back manoeuvre has been started from the same anatomical landmark (for example, the origin of a side branch). The depth and length of calcification, an important determinant of results and complications of coronary interventions, ${ }^{26-28}$ and the spatial geometry of dissections can be better determined. ${ }^{29-32}$

\section{Clinical application}

From the on line and off line analysis of the intravascular ultrasound examination of 52 peripheral and 22 coronary arteries Rosenfield 


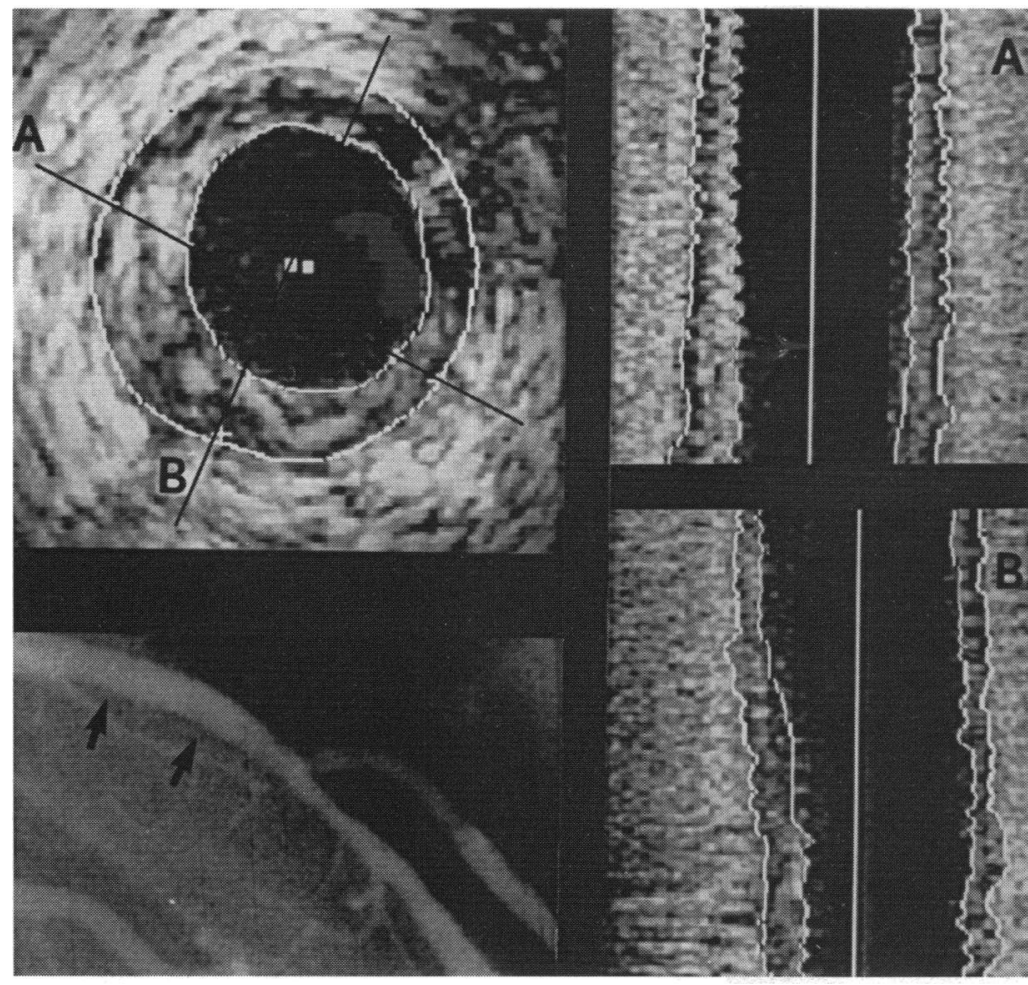

Figure 5 Example of application of the off line reconstruction and analysis program developed at the Thoraxcenter. After detection of the intimal leading edge and the external contour of the vessel in two orthogonal longitudinal views ( $A$ and $B$ ), based on the application of a minimum cost algorithm, the whole set of cross sectional images is processed. For that purpose four individual edge points derived from the longitudinal planes are used, guiding the minimum cost algorithm which defines automatically the contours in all the cross sectional ultrasonic images. The final result can be checked and manual correction may be performed.

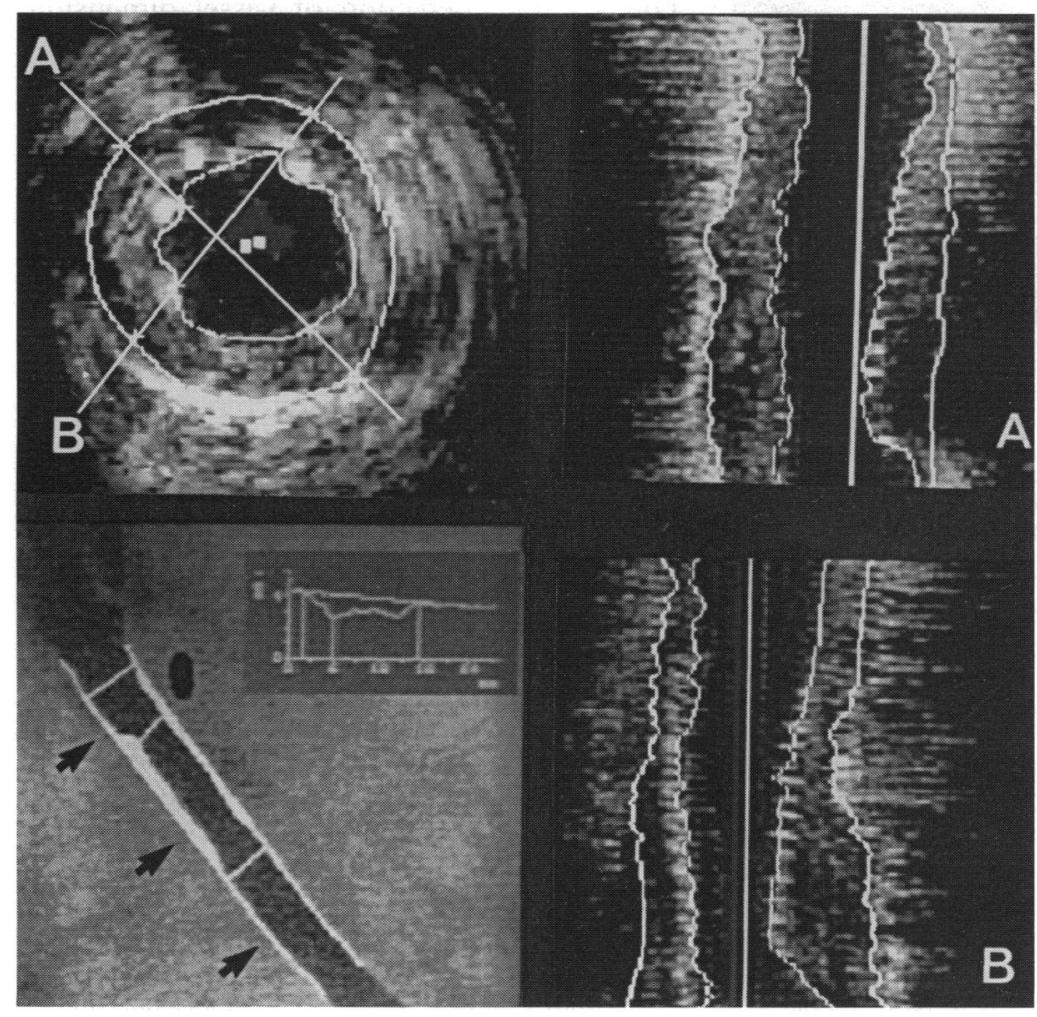

Figure 6 Saphenous vein graft immediately after deployment of a Wallstent (Schneider, Bulach, Switzerland). A previously described custom designed analysis system was used for visualisation and measurement. The length of the vessel segment analysed by three dimensional intracoronary ultrasound is indicated by arrows in the corresponding angiogram (left lower panel). In the longitudinal views the proximal segment of the stented vessel is shown from distal (top) to proximal (bottom). The stent extends from the middle of the segment almost to the lower end of the image, close to the aortic anastomosis of the graft. Due to a huge plaque burden the stent is not fully expanded in its proximal part, suggesting the need for additional intrastent dilatation.

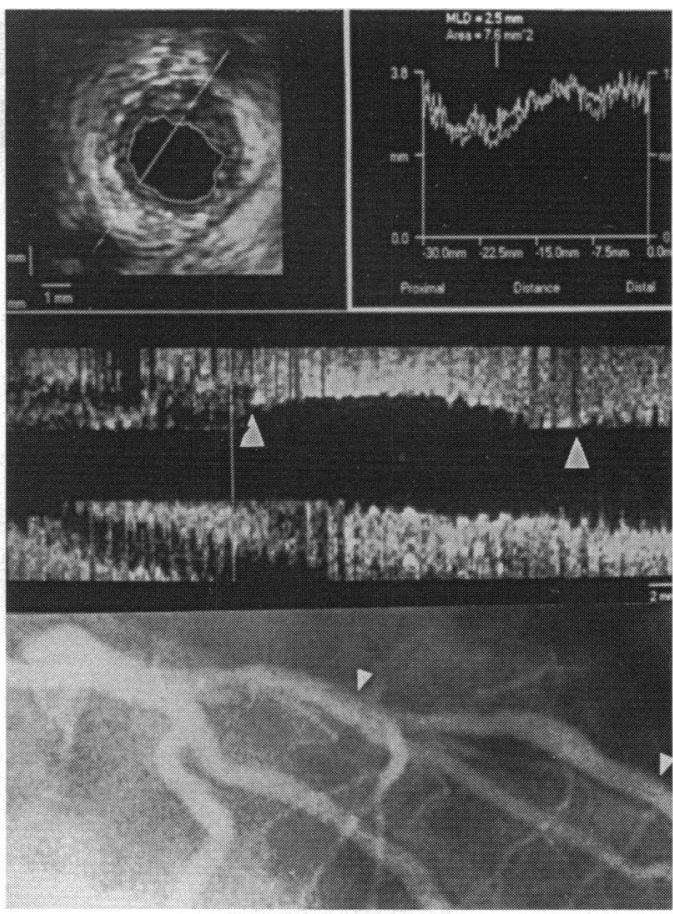

Figure 7 Three dimensional reconstruction after implantation of a $15 \mathrm{~mm}$ Palmaz-Schatz stent (Fohnson and fohnson, Warren, NF, USA) in the mid-segment of a left anterior descending coronary artery (arrowheads in the angiogram, lower panel). The mid-segment of the stent has been overdilated with a short non-compliant balloon. At the proximal and distal end of the stent (indicated by arrowheads in the middle panel), the lumen has a smaller area, matching the area of the diffusely diseased proximal segment (cross sectional image in the upper left panel). Note the precise quantification of the lumen dimensions (cross sectional area and minimum luminal diameter) shown in the upper right panel.

et $a l^{33} 34$ have shown that longitudinal reconstruction facilitates the analysis of dissection and the detection of tunnelling of a false lumen in the recanalisation of total occlusions. Coy et $a l^{35}$ have reported an excellent agreement between three dimensional reconstruction of intravascular ultrasound images and pathological findings in the evaluation of length and depth of post-balloon angioplasty dissection. Recent reports ${ }^{36}{ }^{37}$ have shown the usefulness of computer assisted three dimensional reconstruction in the identification of the true lumen and of the length of dissection before bail-out stenting after coronary angioplasty.

After stenting, three dimensional reconstruction allows the measurement of the longitudinal and radial dimensions of these poorly radiopaque vascular prostheses (figs 4, $6,7)$. The persistence of a moderate residual stenosis inside the stented segment is difficult to detect with angiography, but carries a potentially higher risk of intrastent turbulence leading to subacute thrombosis and long term restenosis. ${ }^{39}$ Goldberg et al have shown that the optimisation of stent deployment with intracoronary ultrasound virtually eliminates the risk of acute or subacute thrombosis ${ }^{40}$ and substantially reduces the late restenosis rate $(24.1 \%$ and $7.9 \%$ without and with intravascular guidance, respectively). ${ }^{41}$ The criteria used for guidance of optimised stent 
implantation can be applied more easily and more reliably to a three dimensional reconstructed image, displaying the entire stented segment and the adjacent reference segments (fig 6).

Intracoronary ultrasound is frequently used for guidance of directional atherectomy. Recent reports have shown that three dimensional reconstruction facilitates the orientation of the cutter in relation to side branches (fig 3) and the detection of deep cuts or spiral cuts from rotation of the atherectomy catheter during plaque removal. ${ }^{42}$ The clinical usefulness of preintervention intracoronary ultrasound in planning and guiding a variety of transcatheter treatments has recently been reported by Mintz et al from Washington, a centre with experience of thousands of ultrasound examinations consistently acquired with a motorised pull back system. ${ }^{43}$ These workers have suggested a specific usefulness of on line three dimensional reconstruction in the evaluation before intervention. ${ }^{43}$

\section{Limitations}

The first critical factor determining the results of the three dimensional reconstruction is the quality of the acquired echographic cross sections. An insufficient delineation of the intimal border or the absence or incomplete circumferential detection of the plaqueadventitia interface precludes automated quantitative measurements of lumen and plaque volumes. Calcium shadowing or intraluminal flaps orientated tangentially to the ultrasound beam may also obscure the underlying wall. ${ }^{44}$ The axial resolution of the current ultrasound probes is reduced in the far field, thus limiting the detection of intimal thickening and of lipid or calcium deposit inside the plaque, ${ }^{45}$ and the lateral and out of plane resolution is limited by the physical dimensions of the piezoelectric crystal. ${ }^{46}$

The use of cross sectional images distorted by the non-uniform rotation of the echographic transducer or by a non-coaxial position of the catheter inside the lumen may create complex artefacts in the reconstructed image.

The presence of a fixed distance between adjacent cross sections is mandatory but difficult to achieve. The use of a motorised pull back or of a sensor measuring catheter displacement cannot completely prevent an uneven speed during the pull back, since bends in the ultrasound catheter may induce a difference between movement of the tip and of the proximal end of the catheter. Another potential source of error is the rotation of the catheter during the pull back, causing a mismatch between the orientation of sequential images. The use of a miniaturised receiving antenna located at the tip of the ultrasound catheter and of an external electromagnetic transmitting antenna in a plane perpendicular to the catheter axis has been proposed as a possible method to measure the orientation of the intracoronary ultrasound catheter. ${ }^{47}$ Curvatures of the vessel also induce a pre- dictable distortion of the three dimensional image which is reconstructed along a straight line through the centre of successive cross sections. Expansion or compression of plaques may result in an overestimation or underestimation of the volumes measured from the reconstructed image. A simultaneous digitised biplane fluoroscopic tracking of the radioopaque transducer and catheter tip has the potential to overcome this limitation but in practice is applicable only for research purposes. ${ }^{48}$ When an accurate reconstruction of the curvature of the vessel is acquired, computer simulation can be applied to reconstruct the arterial flow profile three dimensionally. ${ }^{49}$ These models can obviate the need of a direct measurement of flow velocity in segments with a non-uniform velocity profile (curvatures, stenosis) in which the assessment is difficult also with state of the art intracoronary ultraminiaturised probes. ${ }^{5051}$ The study of flow disturbances is of great interest in evaluating the relationship between location of atherosclerotic plaque and wall stress in vivo and detecting the persistence of alterations in the flow field after interventions which may facilitate wall thrombosis and trigger the restenosis process.

The systolic expansion of the coronary vessel and the movement of the catheter inside the vessel during the cardiac cycle generate a characteristic saw fish appearance of the vessel, more evident in arteries with large motility such as mid-right coronary arteries and bypass grafts. Methods of electrocardiographic gating of the image acquisition can eliminate these artefacts, but require at present a significant increase of the examination time and the complexity of the instrumentation.

\section{Conclusions}

The availability of on line three dimensional reconstruction allows the technique to be applied for guidance and immediate assessment of coronary interventions. High quality cross sectional images are mandatory to achieve an accurate detection of vessel lumen and plaque. Algorithms for blood subtraction and quantitative measurement of lumen and plaque volumes can be applied. Inaccuracies in image acquisition may induce artefacts of the reconstructed image, but our experience indicates that computer generated longitudinal and transverse images derived from the three dimensional image dataset facilitate interpretation and clinical application of intracoronary ultrasound.

1 Hodgson J McB. Intracoronary ultrasound. In: Faxon DP, Holmes DR, Sanborn TA, Schatz RA, eds. Interventional cardiology nevusletter, vol 2-2. New York: Elsevier Science, 1994:12-13.

2 Kitney RI, Moura L, Straughan K. Three-dimensional intravascular ultrasound. In: Bom N, Roelandt JRTC, eds. Intravascular ultrasound. Dordrecht: Kluwer, 1989: eds. Intrav 46.

3 Roelandt JRTC, Di Mario C, Pandian NG, Li W, Keane $\mathrm{D}$, Slager CS, et al. Three-dimensional reconstruction of intracoronary ultrasound images: rationale, approaches, intracoronary ultrasound images: rationale, approaches,

4 Mintz GS, Keller MB, Fay FG. Motorized IVUS transducer pull-back permits accurate quantitative axial meaducer pull-back permits accurate quantitative
surements (abstr). Circulation 1992;86:I-323. 
5 Gussenhoven EJ, van der Lugt A, Van Strijen $M, \mathrm{Li} \mathrm{W}$, Kroeze H, The SHK, et al. Displacement sensing device enabling accurate documentation of catheter tip position. In: Roelandt J, Gussenhoven EJ, Bom N, eds. Intravascular ultrasound 1993. Dordrecht: Kluwer, 1993: 157-66.

6 Weissman NJ, Palacios IF, Weyman AE. Dynamic expansion of coronary arteries. Implications for intravascular ultrasound measurement (abstr). $₹ \mathrm{Am}$ Coll Cardio 1994;23:242A.

7 Roelandt JRTC, Ten Cate FJ, Vletter WB, Taams MA. Ultrasonic dynamic three dimensional visualization of the heart using a vario-plane transesophageal imaging the heart using a vario-plane transesophageal imaging Echocardiogr 1994;7:217-9.

8 Hausmann D, Friedrich G, Sudhir K, Mullen WL, Soni B, Fitzgerald PJ, et al. 3D intravascular ultrasound imaging with automated border detection using $2.9 \mathrm{~F}$ Catheters (abstr). F Am Coll Cardiol 1994;23:174A.

$9 \mathrm{Li} \mathrm{W}$, Gussenhoven WJ, Zhong Y, The SHK, Di Mario C Madretsma S, et al. Validation of quantitative analysis of intravascular ultrasound images. Int $\mathcal{f}$ Card Imaging 1991;6:247-54

10 Di Mario C, The SHK, Madretsma S, van Suylen RJ, Wilson R, Bom N, et al. Detection and characterization of vascular lesions by intravascular ultrasound. An in-vitro
correlative study with histology. $₹ \mathrm{Am}$ Soc Echocardiogr 1992;19:135-46.

$11 \mathrm{Li} \mathrm{W}$, Bosch JG, Zhong Y, von Urk H, Gussenhoven EJ, Mastik F, et al. Image segmentation and 3D reconstruction of intravascular ultrasound images. Acoustic Imaging 1993;20:489-96.

12 Birgelen von C, Di Mario C, Li W, Camenzind E, Ozaki $\mathrm{Y}$, de Feyter PJ, et al. Volumetric quantification in intracoronary ultrasound: validation of a new automatic concoronary ultrasound: validation of a new automatic contour detection method with integ
(abstr). Circulation 1994;90:I-550.

13 Escaned J, Baptista J, Di Mario C, Ozaki Y, Roelandt JRTC, Serruys PW, et al. Detection of coronary atheroma by quantitative angiography: insights gained from intracoronary ultrasound imaging (abstr). $\mathcal{F} A m$ Coll Cardiol 1994;23:174.

14 Ge J, Erbel R, Zamorano J, Koch L, Kearney P, Görge G et al. Coronary artery remodeling in atherosclerotic disease: an intravascular ultrasonic study in vivo. Coronary Artery Dis 1993;4:981-6.

15 Rosenfield K, Kaufman J, Losordo DW, Isner JM. Lumen cast analysis: a quantitative format to expedite on-line analysis of $3 \mathrm{D}$-intravascular ultrasound images (abstr). $\Im$ Am Coll Cardiol 1992;19:115A.

16 Matar FA, Mintz GS, Douek PC, Leon MB, Popma JJ. Three-dimensional intravascular ultrasound: a new stan-
dard for vessel lumen volume measurement? (abstr). dard for vessel lumen volume

17 Gupta M, Connolly AJ, Zhu BQ, Sievers RE, Sudhir K, Sun YP, et al. Quantitative analysis of progression and regression of atherosclerosis by intravascular ultrasound validation in a rabbit model (abstr). Circulation 1992 86:I-518.

18 Galli FC, Sudhir K, Kao AK, Fitzgerald PJ, Yock PG Direct measurement of plaque volume by three-dimen-
sional ultrasound: potential and pitfalls (abstr). $\mathcal{F} \mathrm{Am}$ Coll Cardiol 1992;19:115A.

19 Dhawale P, Rasheed Q Berry J, Hodgson J. Quantification of lumen and plaque volume with ultrasound: accuracy and short term variability in patients (abstr). Circulation 1994;90:I-164.

20 Braden GA, Herrington DM, Downes TR, Kutcher MA, Little WC. Qualitative and quantitative contrasts in the mechanisms of lumen enlargement by coronary balloon angioplasty and directional coronary atherectomy. $\mathcal{F} \mathrm{Am}$ Coll Cardiol 1994;23:40-8.

21 Tenaglia AN, Buller CE, Kisslo KB, Stack RS, Davidson CJ. Mechanisms of balloon angioplasty and directional coronary atherectomy as assessed by intracoronary ultrasound. $₹$ Am Coll Cardiol 1992;20:685-91.

22 Suneja R, Nair NR, Reddy KG, Rasheed Q, Sheehan HM Hodgson JM. Mechanisms of angiographically success ful directional coronary atherectomy. Am Heart $\mathcal{F} 1993$; 126:507-14

23 Di Mario C, Camenzind E, Ozaki Y, Gil R, von Birgelen $\mathrm{C}$, Umans V, et al. Is the mechanism of restenosis device independent? Serial assessment with intracoronary ultrasound (abstr). Circulation 1994:90:I-24

24 Mintz GS, Popma J, Pichard AD, Kent KM, Satler LF, Painter JA, et al. Mechanism of late arterial response to transcatheter therapy: a serial quantitative angiographic and intravascular ultrasound study (abstr). Circulation 1994;90:I-24

25 The GUIDE trial investigators. IVUS-determined predictors of restenosis in PTCA and DCA: an interim report from the GUIDE trial, Phase II (abstr). Circulation 1994;90:I-25.

26 Honye J, Mahon DJ, White CJ, Ramee SR, Wallis JB, AlZarka A, et al. Morphological effects of coronary balloon angioplasty in vivo assessed by intravascular ultrasound angioplasty in vivo assessed by intra

27 Fitzgerald PJ, Ports TA, Yock PG. Contribution of localized calcium deposits to dissection after angioplasty. An observational study using intravascular ultrasound. Circulation 1992;86:64-70.

28 Potkin BN, Keren G, Mintz GS, Douek PC, Pichard AD, Satler LF, et al. Arterial response to balloon coronary angioplasty: an intravascular ultrasound study. $\mathcal{f} \mathrm{Am}$ Coll Cardiol 1992;20:942-51.

29 The SHK, Gussenhoven EJ, Zhong Y, Li W, van Egmond $\mathrm{F}$, Pieterman $\mathrm{H}$, et al. Effect of balloon angioplasty on femoral artery evaluated with intravascular ultrasound imaging. Circulation 1992;86:483-93.

30 Losordo DW, Rosenfield K, Pieczek A, Baker K, Harding $M$, Isner JM. How does angioplasty work? Serial analysis of human iliac arteries using intravascular ultrasound. Circulation 1992;86:1845-58.

31 Werner GS, Sold G, Buchwald A, Wiegand V. Intravascular ultrasound imaging of human coronary arteries after percutaneous transluminal angioplasty: morphologic and quantitative assessment. Am Heart $\mathcal{F} 1991 ; 122$ : logic and 2120 .

32 Tenaglia AN, Buller CE, Kisslo KB, Phillips HR, Stack RS. Intracoronary ultrasound predictors of adverse outcomes after coronary artery
Cardiol 1992;20:1385-90.

33 Rosenfield K, Losordo DW, Ramaswamy K, Isner JM. Three-dimensional reconstruction of human coronary and peripheral arteries from images recorded during two-dimensional intravascular ultrasound examination. Circulation 1991;84:1938-56.

34 Rosenfield K, Kaufman J, Pieczek A, Langevin RE, Razvi S Isner JM. Real-time three-dimensional reconstruction of intravascular ultrasound images of iliac arteries. $A m$ Cardiol 1992;70:412-5.

35 Coy KM, Park JC, Fishbein MC, Laas T, Diamond GA, Adler $\mathrm{L}$, et al. In vitro validation of three-dimensional intravascular ultrasound for the evaluation of arterial injury after balloon angioplasty. $₹ \mathrm{Am}$ Coll Cardiol 1992; 20:692-700.

36 Cavaye DM, White RA, Lerman RD, Kopchock GE Tabbara MR, Cormier F, et al. Usefulness of intravascabbara MR, Cormier F, et al. Usefulness of intravasinduced aortic dissection in dogs and for determining the effectiveness of endoluminal stenting. $\mathrm{Am} \mathcal{F}$ Cardio 1992;69:705-7.

37 Schryver TE, Popma J, Kent KM, Leon MB, Mintz GS Use of intracoronary ultrasound to identify the true coronary lumen in chronic coronary dissection treated with intracoronary stenting. Am $\mathcal{F}$ Cardiol 1992;69: 1107-8.

38 Nakamura S, Colombo A, Gaglione A, Almagor Y, Goldberg SL, Maiello L, et al. Intracoronary ultrasound observations during stent implantation. Circulation 1994; 89:2026-34.

39 Mudra H, Klauss V, Blasini R, Kroetz M, Rieber J, Regar $\mathrm{E}$, et al. Ultrasound guidance of Palmaz-Schatz intracoronary stenting with a combined intravascular ultrasound balloon catheter. Circulation 1994;90: 1252-61.

40 Goldberg SL, Colombo A, Nakamura S, Almagor Y, Maiello L, Tobis JM. Benefit of intracoronary ultrasound in the deployment of Palmaz Schatz stents. $\mathcal{F} A m$ Coll Cardiol 1994;24:996-1003.

41 Goldberg SL, Colombo A, Almagor Y, Hall P, Maiello L Nakamura $S$, et al. Has the introduction of intravascula ultrasound guidance led to different clinical results in the deployment of intracoronary stents? (abstr.) Circulation 1994;90:I-610.

42 Smucker ML, Kil D, Sarnat WS, Howard PF. Is threedimensional reconstruction a gimmick or a useful clinical tool? Experience in coronary atherectomy (abstr) f Am Coll Cardiol 1992;19:115A.

43 Mintz GS, Pichard AD, Kovach JA, Kent KM, Satler LF, Javier SP, et al. Impact of preintervention intravascular ultrasound imaging on transcatheter treatment strategies in coronary artery disease. Am f Cardiol 1994;73: in coronar.

44 Di Mario C, Madrestma S, Linker D, The SHK, Bom N, Serruys PW, et al. The angle of incidence of the ultrasonic beam: a critical factor for the image quality in sonic beam: a critical factor for the image quality in
intravascular ultrasound. Am Heart $\mathcal{F} 1993 ; 125: 442-8$.

45 Peters JC, Wouter EM, Miek G, Havenith JE, Rijsteborgh $\mathrm{H}$, Allard $\mathrm{C}$, et al. Histopathological validation of intracoronary ultrasound imaging. $\mathcal{F} \mathrm{Am}$ Soc Echocardiog 1994;7:230-41.

46 Benkeser PJ, Churchwell AL, Lee C, Abouelnasr DM Resolution limitations in intravascular ultrasound imaging. F Am Soc Echocardiogr 1993;6:158-65.

47 Koch L, Kearney P, Erbel R, Roth Th, Ge J, Brennecke R et al. Three dimensional reconstruction of intracoronar ultrasound images: roadmapping with simultaneously digitised coronary angiograms. IEEE Proc Compu Cardiol 1993:89-91.

48 Aretz HT, Gregory KW, Martinelli MA, Gregg RE, Ledet EG, Haase WC. Ultrasound guidance of laser atherectomy. Int $\mathcal{F}$ Cardiac Imaging 1991;6:231-7.

49 Burrel J, McDonald AH, Rothman MT. 3-D computer visualization of arteries and blood flow-in vitro and in vivo. Comput Cardiol 1994:41-6.

50 Di Mario C, Meneveau N, Gil R, de Jaegere P, de Feyter PJ, Slager CJ, et al. Maximal blood flow velocity in severe coronary stenoses measured with a Doppler guidewire. Limitations for the applications of the continuity equation in the assessment of stenosis severity. $A m$ f Cardiol 1993;71:54-61D.

51 Serruys PW, di Mario C, Kern MJ. Intracoronary Doppler. In: Topol EJ, ed. Textbook of interventional car diology, 2nd ed. Philadelphia: WB Saunders, 1994 1069-121. 